\title{
Intussuscepção Intestinal em Adultos
}

\author{
Intussusception in Adults
}

\author{
BERNARDO HANAN ${ }^{1}$; THOMAS REZENDE DINIZ²; MAGDA MARIA PROFETA DA LUZ ${ }^{3}$; SÉRGIOALEXANDRE DA \\ CONCEIÇÃO ${ }^{3} ;$ RODRIGO GOMES DA SILVA $^{3}$; ANTÔNIOLACERDA-FILHO ${ }^{4}$
}

\author{
${ }^{1}$ Residente de Coloproctologia; ${ }^{2}$ Residente de Cirurgia Geral; ${ }^{3}$ Membro do Grupo de Coloproctologia do Instituto Alfa \\ de Gastroenterologia do HC-UFMG; ${ }^{4}$ Coordenador do Grupo de Coloproctologia do Instituto Alfa de \\ Gastroenterologia do HC-UFMG.
}

\begin{abstract}
HANAN B; DINIZ TR; LUZ MMP; CONCEIÇÃO SA; SILVA RG; LACERDA-FILHO A. Intussuscepção Intestinal em Adultos. Rev bras Coloproct, 2007;27(4): 432-438.

RESUMO: A intussuscepção intestinal em adultos é rara. Ao contrário do que ocorre na faixa pediátrica, é, na maioria das vezes, secundária a uma lesão definida com potencial significativo de malignidade. O objetivo deste estudo é avaliar retrospectivamente os aspectos diagnósticos e terapêuticos da intussuscepção intestinal em adultos. Foram estudados, retrospectivamente, os dados relativos às causas, à apresentação clínica, ao diagnóstico e ao manejo da doença em 16 pacientes, sendo 10 do sexo feminino (62\%) e com média de idade de 49 anos (variação de 19-76 anos). Os principais achados clínicos foram: dor abdominal (100\%), náuseas e vômitos $(62,5 \%)$, massa palpável $(62,5 \%)$, distensão abdominal $(37,5 \%)$ e hemorragia digestiva baixa $(31,25 \%)$. Sete pacientes $(43,75 \%)$ apresentaram quadro agudo, sendo seis por obstrução intestinal (37,5\%). 0 diagnóstico pré-operatório foi firmado em 8 pacientes $(50 \%)$, através de exame clínico associado a ultra-sonografia, tomografia computadorizada, trânsito intestinal ou colonoscopia. Todos os pacientes foram submetidos a tratamento cirúrgico. Em cinco pacientes $(31,25 \%)$ o segmento acometido foi o intestino delgado; em seis $(37,5 \%)$ o íleo terminal e a valva ileocecal e em cinco $(31,25 \%)$ havia acometimento colônico. Causa anatômica patológica foi identificada em 14 pacientes (87,5\%), sendo que oito $(50 \%)$ eram portadores de neoplasias malignas. Os procedimentos de ressecção realizados foram enterectomia (18,75\%), hemicolectomia direita com anastomose primária $(31,25 \%)$ ou ileostomia e fístula mucosa $(\mathbf{1 2 , 5 \%})$, retossigmoidectomia a Hartmann (12,5\%), retossigmoidectomia com anastomose primária $(6,25 \%)$ e colectomia total com anastomose íleo-retal $(6,25 \%)$. Dois pacientes $(\mathbf{1 2 , 5} \%)$ foram tratados com redução sem ressecção. Complicações pós-operatórias ocorreram em 3 pacientes (seroma, abscesso de parede abdominal e arritmia cardíaca). Não houve nenhum óbito. Embora incomum, a intussuscepção deve ser suspeitada em casos de dor abdominal crônica e recorrente ou de obstrução intestinal. A ressecção está indicada na maioria dos casos devido à presença de lesão potencialmente maligna, como causa da invaginação intestinal.
\end{abstract}

Descritores: Intussusception intestinal; obstrução intestinal; neoplasias malignas.

A Intussuscepção intestinal em adultos é uma condição pouco comum, sendo responsável por cerca de 1 a 5\% dos casos de obstrução intestinal em adultos $^{2}$, o que faz com que a maioria dos cirurgiões tenha pouca experiência no seu manejo. Aproximadamente 90\% dos casos são secundários à lesão orgânica, diagnosticada, quase sempre, no per-operatório ${ }^{1,4}$. Com o constante avanço dos métodos de imagem, maior número de casos têm sido diagnosticados antes da in- dicação da cirurgia, que se impõe em todos os casos. Permanecem, entretanto, controvérsias quanto à melhor abordagem cirúrgica, tais como o tipo de ressecção, sua extensão e à possibilidade de redução apenas, sem ressecção ${ }^{2,4}$.

O objetivo do presente estudo é avaliar os aspectos diagnósticos e terapêuticos da intussuscepção intestinal em adultos operados em nossa instituição.

Trabalho realizado pelo Grupo de Coloproctologia e Intestino Delgado do Instituto Alfa de Gastroenterologia do Hospital das Clínicas da Universidade Federal de Minas Gerais, Belo Horizonte/MG.

Recebido em 13/08/2007

Aceito para publicação em 29/09/2007 


\section{MATERIAIS E MÉTODOS}

Dezesseis pacientes com idade igual ou maior que 18 anos portadores de intussuscepção foram atendidos e operados no Hospital das Clínicas da Universidade Federal de Minas Gerais de janeiro de 1997 a fevereiro de 2007. Foram avaliados aspectos relacionados à apresentação clínica, ao diagnóstico e ao tratamento. Os dados referentes à tática cirúrgica e ao exame anátomo-patológico da peça cirúrgica foram analisados de forma a definir a localização topográfica, as causas e a natureza das lesões relacionadas à intussuscepção.

\section{RESULTADOS}

Dos dezesseis pacientes com diagnóstico de intussuscepção operados, dez eram do sexo feminino (62,5\%) com média de idade de 49 anos, tendo variado de 19 a 76 anos. História prévia crônica pertinente ao diagnóstico foi identificada em quatro pacientes (25\%), sendo três portadores de síndrome de Peutz-Jeghers $(18,75 \%)$ e um com melanoma metastático $(6,25 \%)$.

O sintoma mais comum foi dor abdominal, relatada por todos os pacientes. Náuseas, vômitos, diarréia, constipação e hemorragia digestiva estiveram entre os sinais e sintomas mais comumente apresentados (Tabela 1). Sete pacientes $(43,75 \%)$ apresentaram sintomatologia aguda, enquanto os demais tinham manifestações crônicas. Dez pacientes $(62,5 \%)$ tinham massa abdominal palpável no exame clínico. Um paciente apresentava abdome agudo perfurativo com peritonite generalizada.

Radiografias simples de abdome foram realizadas em apenas dois pacientes. Os achados radiográficos foram inespecíficos tais como, a presença de níveis hidroaéreos e de alças intestinais dilatadas. Enema baritado foi realizado em um paciente e falhou na identificação de intussuscepção ileocecal. Esse paciente foi submetido a trânsito intestinal baritado que sugeriu o diagnóstico. Em outro paciente submetido a trânsito intestinal foi identificada intussuscepção jejuno-jejunal a 20 centímetros do ângulo de Treitz. Seis pacientes submeteram-se a ultra-sonografia abdominal que foi capaz de fazer o diagnóstico em cinco deles (acurácia de 83,3\%) (Figura 1). Em três pacientes foi realizada tomografia computadorizada, que foi capaz de diagnosticar dois casos (acurácia de 66,6\%). Dois pacientes foram submetidos à colonoscopia, com obtenção do diagnóstico em um caso (acurácia de 50\%). No outro, o exame foi incompleto devido a dificuldades técnicas. Retossigmoidoscopia foi realizada em dois pacientes e, em ambos, o diagnóstico não foi suspeitado. No total, o diagnóstico pré-operatório foi firmado em oito pacientes (50\%). Nos demais, o diagnóstico foi obtido no intra-operatório.
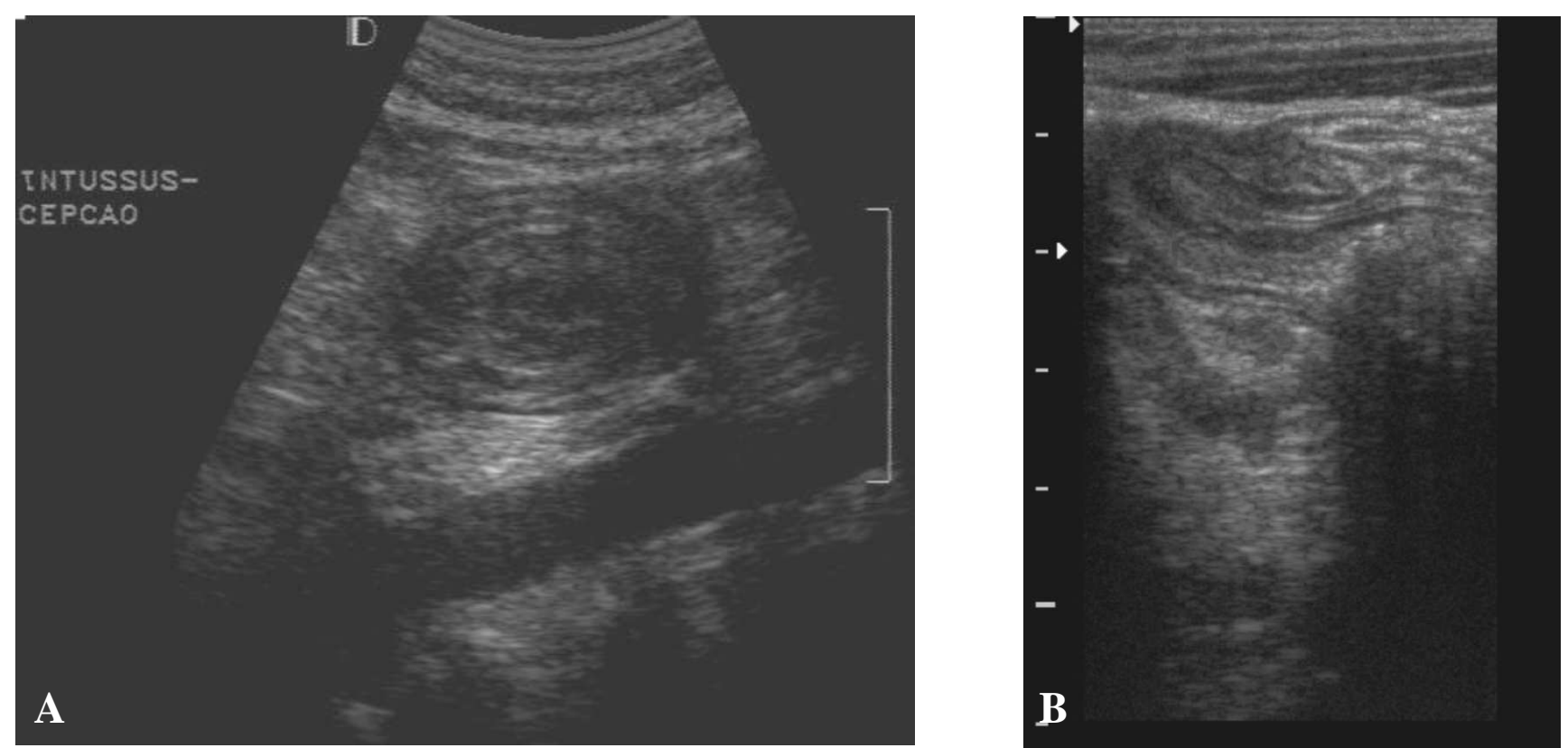

Figura 1 - Ultra-sonografia abdominal em paciente portador de intussuscepção de intestino delgado: A - corte transversal - "Sinal do alvo", e B - corte longitudinal - "Sinal do pseudo-rim" (Cortesia do Dr. Rogério Augusto Pinto e Silva - Departamento de Imaginologia do HC$U F M G)$. 
À laparotomia, cinco pacientes $(31,25 \%)$ apresentavam o intestino delgado acometido, caracterizando intussuscepção jejuno-jejunal em dois casos $(12,5 \%)$ e íleo-ileal em três (18,75\%). Também cinco pacientes $(31,25 \%)$ tiveram o cólon acometido, sendo dois no sigmóide e reto $(12,5 \%)$, um no cólon ascendente $(6,25 \%)$, um no cólon descendente $(6,25 \%)$ e um com intussuscepção de todo o cólon a partir do cólon ascendente até a junção retossigmoidiana $(6,25 \%)$ (Figura 2). Os demais pacientes apresentavam intussuscepção ileocecal (37,5\%) (Figura 3).

Quatorze pacientes $(87,5 \%)$ eram portadores de lesão orgânica responsável pelo quadro de invaginação intestinal, sendo oito (50\%) por neoplasia maligna. Dos pacientes portadores de intussuscepção por lesões benignas, três casos $(18,75 \%)$ foram devidos a hamartomas relacionados à Síndrome de PeutzJeghers, um caso por pólipo adenomatoso gigante $(6,25 \%)$ (Figura 4), um por lipoma (6,25\%) (Figura 5)

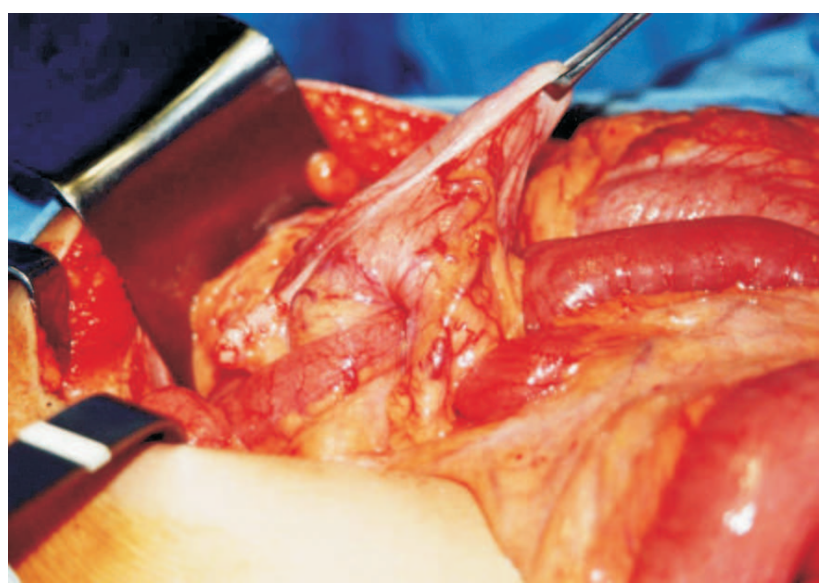

Figura 3 - Imagem de intussuscepção íleo-cecal.

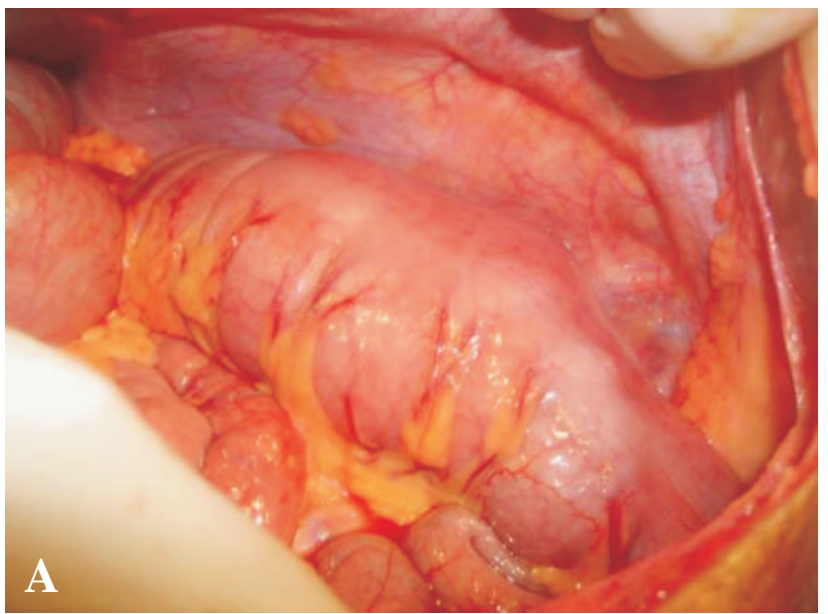

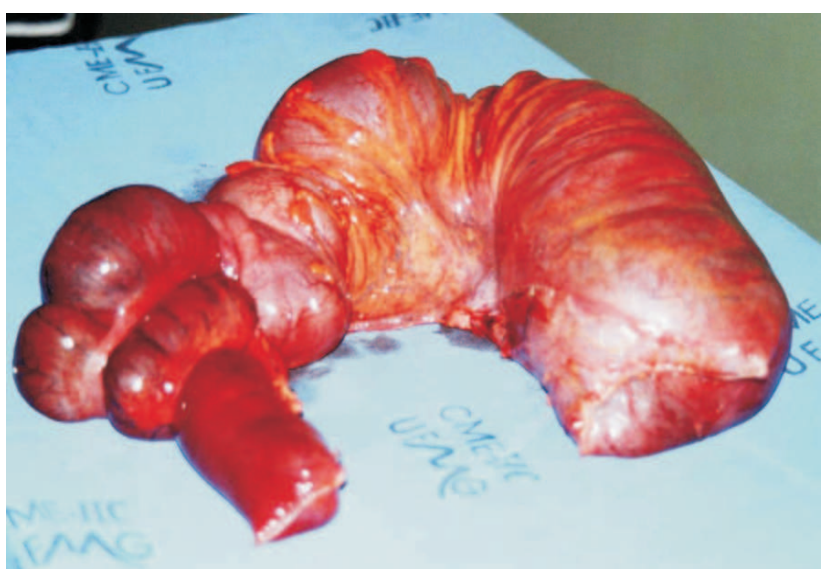

Figura 2 - Imagem de intussuscepção de todo o cólon à partir do cólon ascendente até a junção retossigmoidiana.

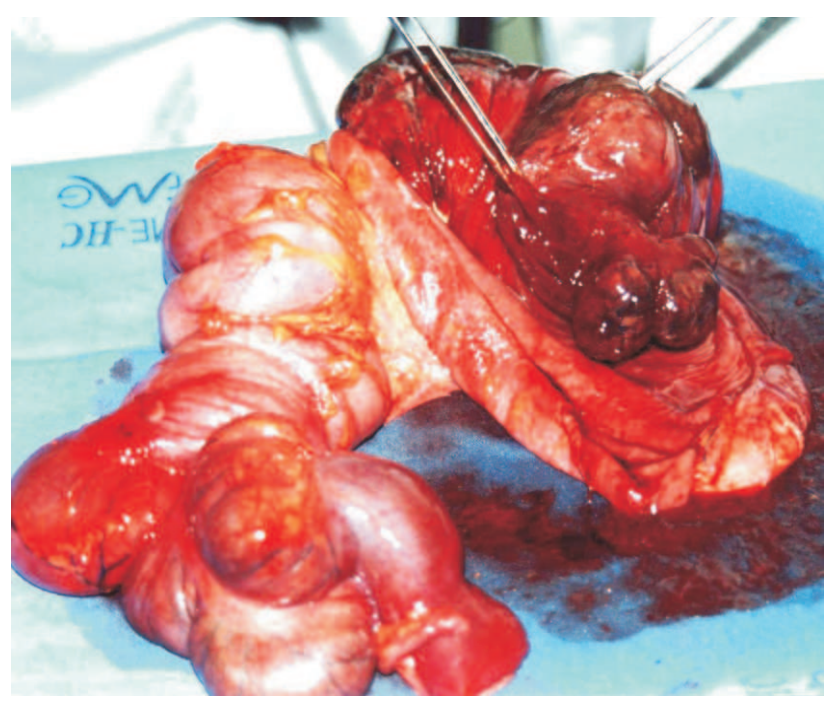

Figura 4 - Imagem de volumoso pólipo adenomatoso causador de intussuscepção colo-cólica.

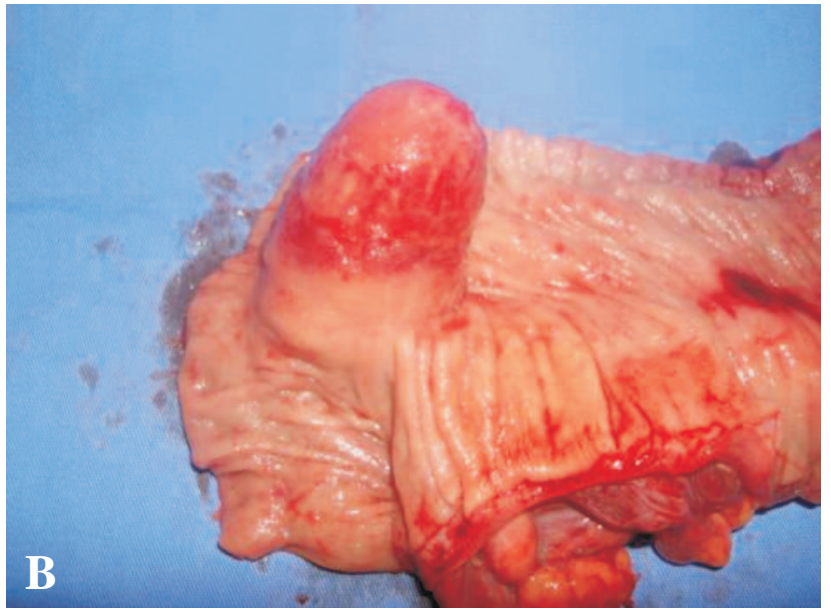

Figura 5 - Imagem de segmento de sigmóide invaginado (A) causado por Lipoma colônico (B). 
e um caso por lesão inflamatória inespecífica $(6,25 \%)$. Dois casos (12,5\%) ocorreram em período pós-operatório, sem identificação de lesões orgânicas associadas (Tabela 2).

Todos os pacientes foram submetidos a tratamento cirúrgico. A tática cirúrgica foi determinada pela localização, pelo tipo de lesão envolvida e pela viabilidade intestinal. Os dois pacientes com intussuscepção pós-operatória de intestino delgado (12,5\%) foram submetidos à redução, sem ressecção, uma vez que não foram identificadas quaisquer lesões relacionadas na palpação per-operatória. Os outros três pacientes com acometimento do intestino delgado $(18,75 \%)$ foram submetidos a enterectomia, sendo três por hamartomas $(18,25 \%)$ e um por melanoma metastático $(6,25 \%)$.

Hemicolectomia direita foi realizada em cinco dos seis pacientes com acometimento do segmento ileocecal $(37,5 \%)$. Em um caso foi realizada hemicolectomia associada à ileostomia e fístula mucosa, devido à perfuração do

Tabela 1 - Apresentação clínica de pacientes adultos portadores de intussuscepção intestinal $(N=16)$.

\begin{tabular}{lcc}
\hline Sintomas & N & \% \\
\hline Dor abdominal & 16 & 100 \\
Náusea e vômito & 10 & 62,5 \\
Massa palpável & 10 & 62,5 \\
Obstrução intestinal & 6 & 37,5 \\
Distensão abdominal & 6 & 37,5 \\
Hemorragia digestiva & 5 & 31,25 \\
Diarréia & 4 & 25 \\
Constipação & 3 & 18,75 \\
Perda de peso & 3 & 18,75 \\
\hline
\end{tabular}

cólon transverso (6,25\%). Esse mesmo procedimento foi realizado em um paciente com síndrome de Peutz Jeghers e múltiplos pólipos com perfuração intestinal (6,25\%). Dois pacientes foram submetidos à colostomia à Hartmann $(12,5 \%)$, um com tumor de cólon descendente e outro de sigmóide. Colectomia subtotal com íleo-reto anastomose e ileostomia protetora foi realizada em um paciente com intussuscepção colo-colônica e isquemia intestinal $(6,25 \%)$ (Figura 2). Retossigmoidectomia foi realizada em um paciente com lipoma volumoso no sigmóide (6,25\%).

Complicações pós-operatórias ocorreram em três pacientes, sendo um caso de arritmia cardíaca, outro de seroma volumoso e um terceiro com abscesso intra-abdominal, conferindo uma taxa de morbidade de $18,75 \%$. O tempo médio de hospitalização foi de sete dias, tendo variado de três a 15 dias. Nenhum óbito ocorreu na presente casuística.

\section{DISCUSSÃO}

A intussuscepção intestinal em adultos é uma entidade que difere em vários aspectos daquela observada na faixa etária pediátrica. Enquanto nas crianças o início dos sintomas geralmente é agudo com dor abdominal em cólica, vômitos, eliminação de fezes com muco e sangue e massa palpável, a apresentação no adulto pode ser subaguda ou crônica. Nas crianças é, na maioria das vezes primária e de natureza benigna, sendo o tratamento realizado com redução com enema baritado em $80 \%$ dos casos. Nos adultos, a doença normalmente é secundária à causa orgânica, sendo de difícil diagnóstico, quase sempre firmado somente durante a laparotomia ${ }^{4,7,11}$.

Essa condição, comum em crianças, é pouco freqüente em adultos ${ }^{2,13,14}$. Estima-se que apenas 5\%

Tabela 2 - Causas e locais de acometimento dos casos de intussuscepção em adultos $(N=16)$.

\begin{tabular}{lccccc}
\hline Causas & & $\mathbf{N}(\boldsymbol{\%})$ & Intestino delgado & Valva ileocecal & Intestino grosso \\
\hline Sem causa orgânica & 2 & $(12,5 \%)$ & 2 & - & - \\
Lesões Benignas & 6 & $(37,5 \%)$ & & & - \\
Inflamação crônica & 1 & $(6,25 \%)$ & - & 1 & 1 \\
Lipoma & 1 & $(6,25 \%)$ & - & - & - \\
Hamartoma & 3 & $(18,75 \%)$ & 2 & 1 & 1 \\
Adenoma & 1 & $(6,25 \%)$ & - & - & 3 \\
Maligna & 8 & $(50 \%)$ & & & - \\
Adenocarcinoma & 7 & $(43,75 \%)$ & - & 4 & - \\
Melanoma & 1 & $(6,25 \%)$ & 1 & - & \\
\hline
\end{tabular}


dos portadores de intussuscepção sejam adultos e que aproximadamente 5\% dos casos de obstrução intestinal em adultos sejam causados por intussuscepção ${ }^{4}$. Em um período de 10 anos foram encontrados apenas 16 casos de intussuscepção em adultos, operados por vários cirurgiões em nossa instituição. Desta forma, mesmo cirurgiões experientes têm oportunidade de acompanhar poucos casos em toda a sua carreira.

Apesar de sua raridade e do diagnóstico ser firmado, muitas vezes, somente à laparotomia, todo cirurgião deve conhecer os aspectos concernentes a essa entidade. Os sinais e sintomas variam entre os diferentes casos e não são específicos. Obstrução intestinal não está presente em muitos casos, sendo que manifestações crônicas e semi-obstrução intestinal são mais comumente observadas $^{2,11}$. No presente estudo, sete pacientes $(43,75 \%)$ apresentaram-se ao Serviço de Pronto Atendimento de nossa instituição com sintomatologia aguda, sendo seis por obstrução intestinal $(37,5 \%)$. Outros nove pacientes apresentavam sintomatologia crônica $(56,25 \%)$.

O sintoma mais comum foi a dor abdominal, presente em $100 \%$ dos casos, o que está de acordo com a literatura ${ }^{4,13,20}$. Os outros sintomas mais comumente observados foram náuseas, vômitos, hemorragia digestiva, alteração do hábito intestinal, distensão e massa abdominal palpável. Todo paciente com esta sintomatologia deve ter, obrigatoriamente, a intussuscepção no espectro dos diagnósticos diferenciais ${ }^{11,17}$. Entretanto, massa abdominal não é um sintoma comum da intussuscepção em adultos, ocorrendo em 7 a $42 \%$ dos $\operatorname{casos}^{2,4}$, o que está em desacordo com o presente estudo, no qual evidenciou-se a presença de massa abdominal na maioria dos pacientes (62,5\%).

O diagnóstico de certeza é baseado nos achados cirúrgicos. Contudo, exames de imagem e procedimentos minimamente invasivos podem ser úteis ${ }^{2,4,14}$. Radiografias simples, com contraste, ultra-sonografia, tomografia computadorizada, endoscopia, e colonoscopia podem revelar o segmento acometido pela doença. A radiografia simples de abdome pode mostrar sinais de obstrução, e estimar sua topografia ${ }^{6,7}$. Estudos baritados podem auxiliar o diagnóstico, porém são contra-indicados nos casos de suspeita de isquemia ou perfuração intestinal. A colonoscopia pode ajudar nos casos de obstrução colônica. Em nossa revisão, dois pacientes submeteram-se à colonoscopia que confirmou o diagnóstico em apenas um dos casos.

A ultra-sonografia é um exame com boa acurácia diagnóstica, tanto em adultos, quanto em cri- anças $^{5,8}$. É de fácil realização, reprodutível e não invasivo ${ }^{6}$. Permite o diagnóstico rápido em mãos experientes capazes de identificar o "sinal do alvo" na visão transversa e o "sinal do pseudorim" na visão longitudinal, bastante sugestivos desta condição ${ }^{5,18}$ (Figura 1). As limitações do método são: a obesidade, a presença de distensão abdominal e a dependência da experiência do examinador. Seis pacientes foram submetidos à ultra-sonografia na presente série, sendo que em cinco deles o diagnóstico foi confirmado por este método.

Também a tomografia computadorizada (TC) tem sido utilizada para avaliar pacientes com obstrução intestinal e suspeita de intussuscepção, sendo uma das armas mais importantes no diagnóstico pré-operatório desta condição ${ }^{3,9,15}$. A densidade da massa gerada pelo segmento acometido, associada ao edema de parede intestinal e mesentérico, origina um sinal característico na TC, o também chamado "sinal do alvo",3,4,7,12. Contudo, a tomografia não é confiável na diferenciação entre neoplasia e espessamento inespecífico da parede intestinal. Além disso, esse exame ainda tem limitações como sua relativa indisponibilidade em todos os serviços de urgência e a necessidade de administração de contraste. No presente estudo, três pacientes foram submetidos à TC, e dois deles tiveram o diagnóstico de intussuscepção confirmado.

A taxa de confirmação diagnóstica pré-operatória descrita na literatura varia aproximadamente de 40 a $50 \% \%^{7,10,14,19}$. Isto está de acordo com nossa observação, na qual oito dos dezesseis pacientes (50\%) tiveram seu diagnóstico firmado previamente à laparotomia. Entretanto, há que se ressaltar que esse percentual poderia ser mais elevado, caso houvesse uma maior disponibilidade dos exames de imagem, sobretudo no ambiente das urgências.

A presença de lesão orgânica tem sido descrita em 70 a $90 \%$ dos casos de intussuscepção em adul$\operatorname{tos}^{2,4,16}$. Dessas, mais de $50 \%$ são malignas ${ }^{1,2,4}$, exatamente como observado na presente casuística, na qual observamos neoplasia em oito de 14 lesões orgânicas identificadas (57\%). Geralmente, as lesões do intestino delgado são benignas, sendo apenas $30 \%$ delas malignas, primárias ou metastáticas. Já no cólon, as lesões malignas representam $66 \%$ dos $\operatorname{casos}^{4,7,15}$. Observou-se que sete dos onze pacientes $(63,6 \%)$ da presente série com acometimento do intestino grosso e um dos cinco (20\%) pacientes com intussuscepção do intestino delgado tinham, como causa orgânica, uma doença maligna. 
Nos adultos, o mecanismo exato da intussuscepção é desconhecido, porém, acredita-se que a lesão presente na parede intestinal altera o peristaltismo normal iniciando o processo de invaginação ${ }^{4,15}$. O mecanismo da intussuscepção idiopática ainda não é compreendido, acontecendo em 8 a $20 \%$ dos casos em adultos, principalmente no intestino delgado. Em dois de nossos casos (12,5\%), a intussuscepção não relacionada a lesões orgânicas ocorreu em período pós-operatório, não tendo sido identificadas condições anatômicas que justificassem o quadro. Considera-se que a presença de aderências seja a principal causa de intussuscepção pós-operatória ${ }^{21}$.

O tratamento padrão da intussuscepção em adulto ainda não está definido. A maioria dos autores concorda que a laparotomia é mandatória na intenção de identificar uma lesão orgânica, muitas vezes, de natureza neoplásica. As controvérsias giram em torno da necessidade e da extensão da ressecção. A possibilidade teórica de implante de células malignas de uma lesão neoplásica leva muitos autores a indicar a ressecção em bloco da lesão, enquanto outros alertam sobre o risco de ressecção intestinal desnecessária ${ }^{2,4}$. Salvo algumas exceções, nos casos de intussuscepção colo-colônica deve-se ressecar o segmento em bloco com finalidade oncológica pelo grande risco de malignidade $^{2,4,7,11}$, o que pôde ser observado no presente estudo, no qual obteve-se um percentual de $63,6 \%$ de lesões malignas no intestino grosso. A presente revisão também identificou lesões malignas em quatro dos oito pacientes com intussuscepção ileocecal (50\%), demonstrando que nesses casos, também a ressecção oncológica em monobloco é a mais indicada.

No caso das lesões do intestino delgado, ao contrário, mais de $60 \%$ são benignas, incluindo lesões inflamatórias, estromais e aderências ${ }^{2}$. Assim sendo, a tentativa de redução inicial é racional, principalmente quando um segmento longo é acometido. Isto porque, a ressecção desnecessária pode ser responsável pelo desenvolvimento de síndrome do intestino curto. Atenção especial deve ser dada para sinais de isquemia, perfuração ou malignidade que contra-indiquem a redução exclusiva ${ }^{11}$. Nos casos de aderências, divertículos e pólipos benignos, o tratamento deve ser individualizado. Lise de aderências, diverticulectomia e polipectomia podem ser usados em casos selecionados $2,7,11$.

\section{CONCLUSÃO}

A intussuscepção em adultos é uma condição que desafia o cirurgião, tanto em relação ao diagnóstico, quanto à abordagem adequada. $\mathrm{O}$ diagnóstico é difícil pelo baixo grau de suspeição, associado à sintomatologia subaguda $\mathrm{e}$ inespecífica. É quase sempre associada a uma lesão orgânica e requer tratamento cirúrgico. Ressecções oncológicas em monobloco devem ser realizadas nos casos de acometimento colônico e ileocecal pelas possibilidades de malignidade e de perfuração. Nos casos de intussuscepção do intestino delgado, a simples redução pode ser suficiente.

ABSTRACT: Intestinal intussusception in adult patients is a rare entity. Differently from pediatric patients, this condition is often secondary to a defined lesion. A significant percentage of these lesions are malignant. Therefore, an optimal treatment is necessary to this condition. The aim of this study is to determine causes and management of adult intussusception. We carried out a retrospective review of adults patients with diagnosis of intussusception from 1997 to 2007 in our institution. Details concerning presentation, diagnosis, management, and pathology were analyzed. Sixteen patients with intestinal intussusception underwent laparotomy. There were 10 women with median age of 49 years (range, 19-76). Abdominal pain was the most common clinical finding. Seven patients (46.6\%) presented with acute symptoms, six caused by intestinal obstruction. Diagnosis of gastrointestinal intussusception was correctly done preoperatively in 8 patients $(50 \%)$. Five patients $(31.25 \%)$ had the lead point of the intussusception in the small bowel, two in the jejunum and three in the ileum. Six patients $(37.5 \%)$ had the lead point in the ileocecal valve resulting in ileocolic intussusception and five patients (31.25\%) had colonic leading points. An anatomic or pathologic cause of the intussusception was identified in 14 patients (87.5\%). In two patients $(\mathbf{1 2 . 5 \%})$ the intussusception occurred in the postoperative period without associated lesions. Malignant neoplasms accounted for eight of sixteen patients $(50 \%)$. All patients underwent operative management. The two patients who have postoperative intussusception of the small bowel underwent reduction with no resection. The others three patients with small bowel intussusception underwent to enterectomy. Right hemicolectomy was performed in five of the six patients with ileo-colonic intussusception. Retossigmidectomy was performed in one of the two patients with sigmoid intussusception. Hartmann's procedure was performed in two patients. Subtotal colectomy with ileorectal anastomosis and ileostomy was performed in one patient with colo-colonic intussusception. Minor postoperative complications occurred in 3 patients. Although uncommon, surgeons need to be aware about epidemiology and treatment options for adult intussusception. The symptoms and signs are often non-specific and the surgeon might be faced with the diagnosis only at laparotomy. Surgical resection is indicated in most of the cases due to the possibility of malignant lesion as cause of intussusception.

Key words: Intestinal obstruction; intestinal intussusception; malignant neoplasm. 


\section{REFERÊNCIAS}

1. Akcay MN, Polat M, Cadirci M, Gencer B. Tumor induced ileoileal invagination in adults. Am Surg 1994;60:980-981.

2. Azar T, Berger DL. Adult intussusception. Ann Surg 1997;226:134-138.

3. Bar-Ziv J, Solomon A. Computed tomography in adult intussusception. Gastrointest. Radiol. 1991;16:264-266.

4. Begos DG, Sandor A, Modlin I. The diagnosis and management of adult intussusception. Am J Surg 1997;73:88-94.

5. Boyle MJ, Arkell LJ, Williams JT. Ultrasonic diagnosis of adult intussusception. Am J Gastroenterology 1993;88:617618.

6. Cerro P, Magrini L, Porcari P, De Angelis O. Sonographic diagnosis of intussusception in adults. Abdom Imaging2000;25:43-47.

7. Eisen LK, Cunningham JD, Aufses AH Jr. Intussusception in adults: institutional review. J Am Coll Surg 1999;88:25-28.

8. Fuji Y, Taniguchi N, Itoh K. Intussusception. Induced by villous tumor of the colon: sonographic findings. J Clin Ultrasound 2002;30:48-51.

9. Gayer G, Apter $\mathrm{S}$, Hofmann $\mathrm{C}$ et al. Intussusception in adults: CT diagnosis. Clin. Radiol. 1998; 53: 53-57.

10. Hurwitz LM, Gertler SL. Colonoscopic diagnosis of ileocecal. Gastrointest Endosc 1986;32:217-218.

11. Martin-Lorenzo JG, Torralba-Martinez A, Liron-Ruiz R et al, Intestinal invagination in adults. Int $\mathrm{J}$ Colorectal Dis 2004;19:68-72.

12. Merine D, Fishman EK, Jones B, et al. Enteroenteric intussusception: in nine patients. Am J Roentgenol 1987;148:1129-1132.
13. Nagorney DM, Sarr MG, Mcilrath DC. Surgical management of intussusception in the adult. Ann Surg 1981;193:230-236.

14. Reijnen H, Joosten H, de Boer H. Diagnosis and treatment of adult intussusception. Am J Surg 1989;158:25-8.

15. Takeuchi K, Tsuzuki Y, Ando T et al. The diagnosis and treatment of adult intussusception. J. Clin. Gastroenterol. 2003; 36:18-21.

16. Thomas AW, Mitre R, Brodmerkel GJ. Sigmoido-rectal intussusception due to sigmoid lipoma. J Clin Gastroenerol 1995;21:257.

17. Warshauer DM, Lee JKT. Adult intussusception detected at CT or MR imaging: clinical-imaging correlation. Radiology 1999;212:853-860

18. Weilbaecher D, Dolin JA, Hearn D, Ogden W. Intussusception in adults. Am J Surg 1971;21:531-535

19. Weissberg DL, Scheibe B, Ineopold GR. Ultrasonic appearance of adult intussusception. Radiology 1987;30:525-530.

20. Zissin R, Gayer G, Konen O, Shapiro-Feinberg M. Transient colocolic intussusception. J Clin Imaging 2000;24:8-9.

21. Zubaidi A, Al-Saif F, Silverman R, Adult Intussusception: A Retrospective Rewiew. Dis Colon Rectum 2006;49:1546-1551.

\section{Endereço para correspondência:}

PROF. DR. ANTÔNIO LACERDA-FILHO

Instituto Alfa de Gastroenterologia

Hospital das Clínicas da UFMG - $2^{\circ}$ andar

Av. Alfredo Balena, 110

Belo Horizonte - MG - BRASIL

CEP 30130-100

Tel: 55-31-32489403

Fax: 55-31-32489251

E-mail: alacerda@ufmg.br 